\title{
A Closed-form formula for RSSI-based DoA Estimation with Switched Beam Antennas
}

\author{
Stefano Maddio ${ }^{1}$, Marco Passafiume ${ }^{2}$, Alessandro Cidronali ${ }^{3}$, Gianfranco Manes ${ }^{4}$ \\ Dept. of Information Engineering, University of Florence, V.S. Marta, 3, I-50139, Florence, Italy \\ $\left\{{ }^{1}\right.$ stefano.maddio, ${ }^{2}$ marco.passafiume, ${ }^{3}$ alessandro.cidronali, ${ }^{4}$ gianfranco.manes $\} @$ unifi.it
}

\begin{abstract}
In this paper we present a novel Direction of Arrival (DoA) estimator for Switched Beam Antennas (SBA). The proposed approach is based on the best analytic approximation of the power pattern ratio between adjacent beams.

Under quite general assumptions, the proposed approach resolves to the solution of a quadratic equation. Compared to conventional estimators, the precision of the proposed approach is competitive, permitting a cost-effective solution for low complexity implementation. Computer simulations and experimental verifications in a anechoic setup confirm the proposed approach when operating with a circular polarized SBA and the received signal strength data collected with a low-cost commercial transceiver.
\end{abstract}

A mean error of $1.1^{\circ}$ with a standard deviation of $1.6^{\circ}$ is experimentally observed when the SBA communicates with a co-polarized target at the range of 2 meters. Only 5\% of the observation angle is affected by an error exceeding $5^{\circ}$.

Keywords-Indoor positioning system, Switched beam antenna (SBA), Direction of arrival (DoA), Received Signal Strength Indicator (RSSI).

\section{INTRODUCTION}

In recent years, the interest of the scientific community in the topic of indoor positioning is in constant growth. In particular, the solution based on simple and cost effective Component-Off-The-Shelves (COTS) are becoming the key technology to permit pervasive location based services directly available to the final user, enabling context-aware applications [1], [2].

Switched Beam Antennas (SBA) are advantageous devices in the fields of mobile communications devices. Capable of a predetermines set of beams, SBA's combine the advantageous feature of adaptive beamformers, such as robustness to multi-path increased coverage, and resistance to signal interception and interference, to simple structure and hardware.

In recent literature, it was demonstrated that a node designed around legacy WiFi transceivers can effectively estimate the direction of arrival working in unmodified IEEE 802.11 protocol, simply operating with the Received Signal Strength Indicator (RSSI), the simple signal power meter embedded in all commercial transceiver [3], [4]. The enabling technology for this goal is the Space Division Multiple Access (SDMA) mechanism enabled by SBAs.

Various canonic algorithms can be adapted to this architecture, such as least square estimator [5], MUSIC or [6], and many others. The estimated DoAs from a network of devices can subsequently applied to multi-angulation technique resulting in the absolute positioning.
In this paper we present and demonstrate a very simple approach for the DoA estimation based on the best analytic approximation of the power pattern of a SBA. This approximation leads to a estimation formula of extremely low complexity, hence suitable to implemented in simple embedded hardware with reduced computational capabilities. The validity of this estimation is conditional to the actual arrangement of the SBA, but it is quite relaxed for the canonical regular Uniform Circular Array (UCA) arrangement.

In order to explain our solution, this paper is organized as follows: Section II describes the target system for this approach, and in section III the algorithm is proposed. Section IV demonstrates the approach with a set of full-wave simulation, and Section $\mathrm{V}$ presents the actual measured performances obtained in anechoic setup. Section VI finishes the proposed work dawning some conclusions.

\section{Switched BEAm Antennas}

Switched Beam Antennas (SBA) are smart radiators capable of set of predetermined beam selected by an external control. The simplest form of SBA consists in an array of $\mathrm{N}$ independent elementary radiators pointing toward different directions, fed by a passive recombining network such as the butler matrix, or directly fed by a non-reflective Single Pole $\mathrm{N}$ Through (SPNT) RF switch connected to the transceiver.

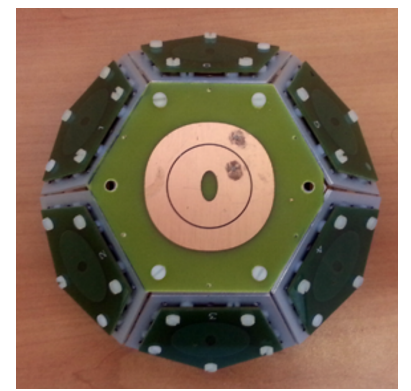

(a) Top view photo

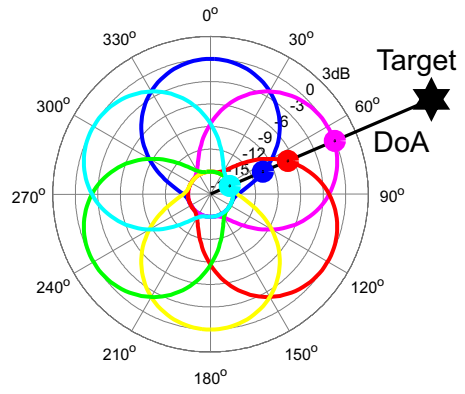

(b) Observation model scheme
Fig. 1: Concept of the proposed localization algorithm applied to an hexagonal-based SBA.

In general, the arrangement of the antenna elements, and hence the radiation beams, has a great impact on the performance of the localization system (as explained in [5], [7], [8]). Upon the reception of a radio message, the beams should provide angular uncorrelated signals to maximize the multiplexing effect, while covering the entire angular range in the cumulative sense to avoid nulls in the communication - see 
Fig. 1b. In addition, SBAs operating in Circular Polarization (CP) increase the reception robustness due to their intrinsic multi-path rejection capability [9], [10], making the RSSI data much less noisy [5].

In the following sections we refer to SBA arranged as an Uniform Circular Array (UCA), equipped with the commercial System-On-Chip (SOC) CC2430, from Texas Instruments. This SOC is complaint with IEEE 802.15.4 and ZigBee operation, and it is equipped with the efficient, while computational limited, 8051 industrial controller. The built-in RSSI module, directly available to the end user, operates averaging the impinging signal power over an 8 symbols periods $(128 \mu \mathrm{s})$. The RSSI data is a 8-bit formatted data register related to the actual incoming power in $\mathrm{dBm}$ through an calibration equation provided by the manufacturer, with a resolution of $1 \mathrm{~dB}$.

\section{ALGORITHM}

In this section the algorithm and its implementation is proposed. To fix the idea, an SBA made of patch antennas operating in their fundamental mode is considered. For this class of antennas, the radiation pattern can be analytically modeled as [11], [5]:

$$
G_{n}(\theta)=\left(\frac{1+\cos \left(\theta-\theta_{n}\right)}{2}\right)^{m}
$$

where $\theta_{n}=2 \pi n / N$, is the pointing angle of each antenna, with $\mathrm{N}$ number of the antennas, and $m$ is the exponent modeling the Half Power Beam Width (HPBW) according to:

$$
m=\frac{1}{\log \left(1+\cos \left(\theta_{H P}\right)\right)-1}
$$

where $\theta_{H P}$ is the HPBW expressed in radians. Note that the analytic expression in (1) is an ideal approximation valid only around the gain maximum, since important characteristics of the pattern, such as front to back ratio, are ignored in this model. In addition, the interaction of closed packed antennas deforms the pattern, with additional un-idealities (see Fig. 3). Nevertheless it can be applied also to arrays as long as the principal beam regular enough as in [12].

For an unobstructed line of sight scenario, considering a signal emitted by a single tag from the unknown direction $\theta$, the sequential collection of the power impinging on the SBA resolves into a N-dimensional RSSI vector according to the following observation model:

$$
\operatorname{RSSI}_{n}(k)=G_{n}^{d B}(\theta)+P_{0}^{d B m}+w(k) ;
$$

where $P_{0}^{d B m}$ is the impinging power at the SBA section expressed in $\mathrm{dBm}, G_{n}^{d B}(\theta)$ is the gain of the n-th antenna for direction $\theta_{0}$ expressed in $\mathrm{dB}$ form, $\mathrm{w}$ is a white Gaussian noise and $\mathrm{k}$ is a time (repetition) index. In the following, the $\mathrm{k}$ index is suppressed to indicate a mean quantity over the observation time, therefore model in (3) recasted in vector form is:

$$
\mathrm{RSSI}=\mathbf{G}^{\mathrm{dB}}(\theta)+\mathbf{P}_{\mathbf{0}}^{\mathrm{dBm}}
$$

To solve (4), well-known methods like MUSIC [6] and similar spectral manipulation are based on the robust comparison of the latter with the stored gain pattern [5]. However, these approaches imply complex algebraic operations, not suitable for low complexity hardware such as the $8051 \mu$-controller.
Another approach is followed here, inspired to the one presented in [13]. First of all, the RSSI set is sorted in descending order, and the corresponding sorting of the beam domains is considered. Hereinafter the RSSI vector is considered as the sorted one, to avoid redundancy of formalism, along with the linear-form $r=10^{0.1 \mathrm{RSSI}}$. Therefore $r_{1}$ is the maximum received power, expressed in $\mathrm{mW}$, and the DoA to be estimated is expected to be found in the range:

$$
\phi=\left[\phi_{1}-\Delta, \phi_{1}+\Delta\right]
$$

With $\Delta=\pi / N$, having supposed identical antennas, hence identical beam-widths [13].

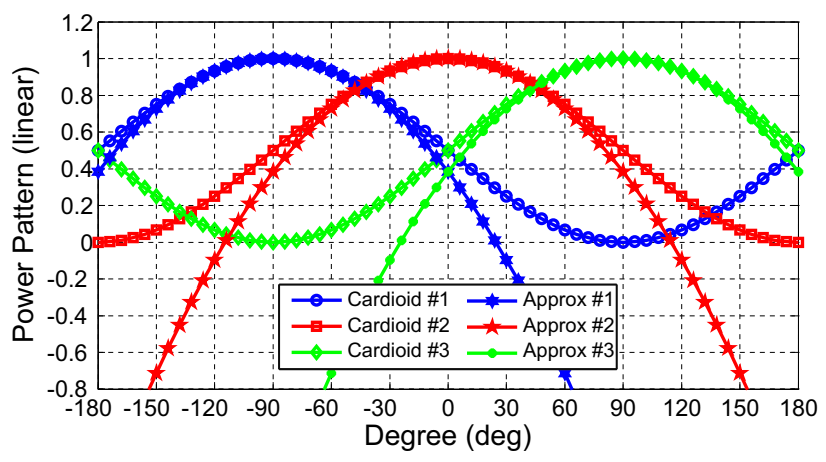

Fig. 2: Comparison between a set of three mathematical cardioid shapes described by (1) and their Taylor approximation according to (7).

At this point, instead of operating on the original expression or find the correlation of the RSSI vector, (3) is simplified applying some basic rules of calculus. The gain expression in (1) - which is expressed in linear form - can be substitute by the Taylor expansion around each beam maximum:

$$
G_{n}(\theta) \doteq\left(1-\left(\frac{\theta-\theta_{n}}{2}\right)^{2}\right)^{m}
$$

Which can be further simplified in

$$
G_{n}(\theta) \approx 1-\frac{m}{4}\left(\theta-\theta_{n}\right)^{2}
$$

Therefore (7) permits to write a simplified equality starting from the collection of RSSI.

In particular, considering \#1 and \#2 from the sorted RSSI vector, and neglecting the noise term after the time mean, (3) can be rewritten in linear form:

$$
\begin{aligned}
& r_{1}=P_{0} G_{1}(\theta)=P_{0}\left(1-\frac{m}{4}\left(\theta-\theta_{1}\right)^{2}\right) \\
& r_{2}=P_{0} G_{2}(\theta)=P_{0}\left(1-\frac{m}{4}\left(\theta-\theta_{2}\right)^{2}\right)
\end{aligned}
$$

Where $P_{0}$ is the linear $(\mathrm{mW})$ form of $P_{0}^{d B m}$. Rearranging, the following equation, which is independent from $P_{0}$, is derived:

$$
r_{1}\left(1-\frac{m}{4}\left(\theta-\theta_{2}\right)^{2}\right)=r_{2}\left(1-\frac{m}{4}\left(\theta-\theta_{1}\right)^{2}\right)
$$

In this expression, the unknown value $\theta$ is the only variable, consider that $\theta_{1}, \theta_{2}$ and $m$ are system constant, while $r_{1}$ and $r_{2}$ are collected after each cycle of RSSI reading. 
The manipulation of (10) leads to a trivial quadratic equation:

$$
\theta^{2}-2 \frac{r_{1} \theta_{2}-r_{2} \theta_{1}}{r_{1}-r_{2}} \theta+\frac{r_{1} \theta_{2}^{2}-r_{2} \theta_{1}^{2}}{r_{1}-r_{2}}+\frac{4}{m}
$$

which can be solved with the classical rule form calculus, The validity of this solution is subjected to the validity of the substitution in (7), which in turn only holds in the angular range where two antennas are concurrent within their principal lobes, where the simplified shape fits very well the actual behavior, as demonstrated in Fig. 2. This implies that the proposed approach works well when there is enough pattern overlapping, which is the common case for SBAs.

\section{Simulation Results}

The proposed approach is tested with a set of simulation obtained with a full-wave CAD. Three kinds of SBA are implemented as real-life device, and a target antenna is implemented as well. In each case the test antenna orbits around the SBA under test. The simulated transmission coefficients between the probe antenna and each port of the SBA is recorded. The ordinated set or them with the addition of white Gaussian noise and truncated to simulate the $1 \mathrm{~dB}$ resolution of RSSI embedded in commercial transceivers [4].

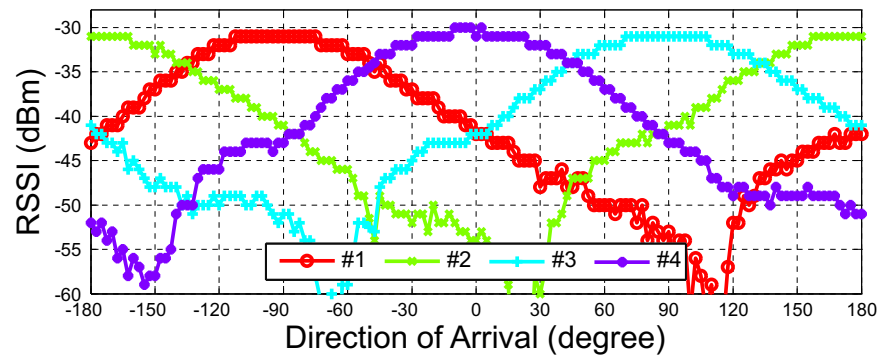

(a) Four beams case

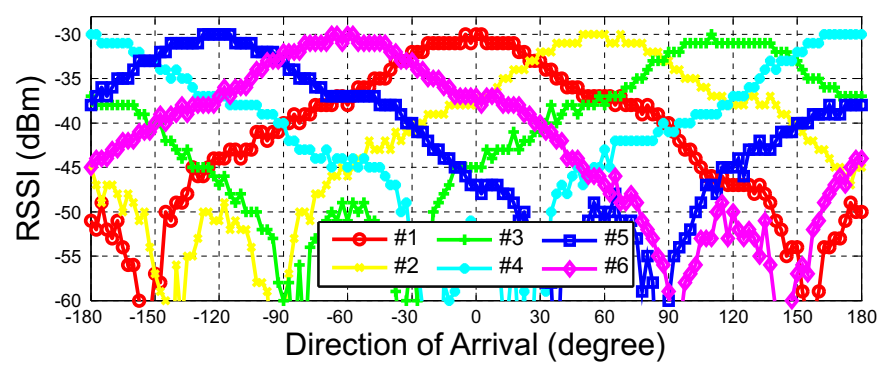

(b) Six beams case

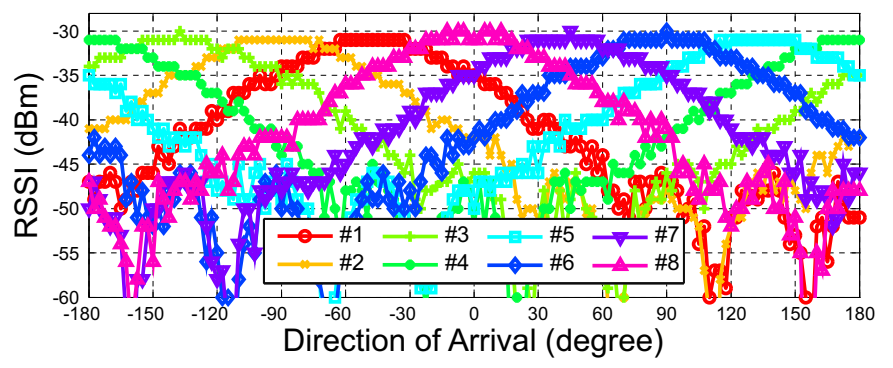

(c) Eight beams case

Fig. 3: Collected RSSI for three simulation cases.
Figure 3 depicts the collected data for the case $\mathrm{N}=$ 4,6,8. A full-wave CAD is able to predict the different slopes of each beam in response to the different mutual coupling because of different mechanic arrangement, making this model effective to predict the actual experimental behavior. As a consequence, great care has to be used identifying the actual exponent in (7), and its consequently in (10). In addition, the maximum gain of each beam can be different. It is possible to take care of all non-idealities, making (10) more accurate, applying a calibration procedure as the one in [14]. The computational complexity is not affected by this procedure: the only difference for the algorithm is to store a table of system constants, composed of no more than $2 \mathrm{~N}$ values.

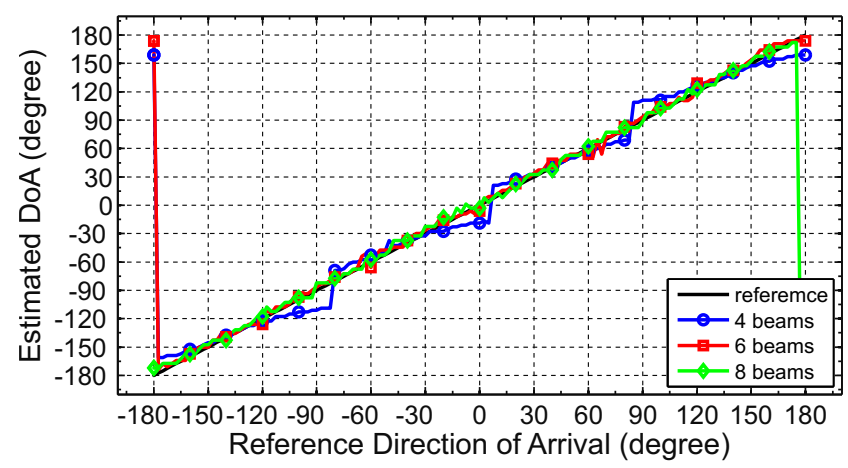

(a) Estimation error along the entire $360^{\circ}$ angle

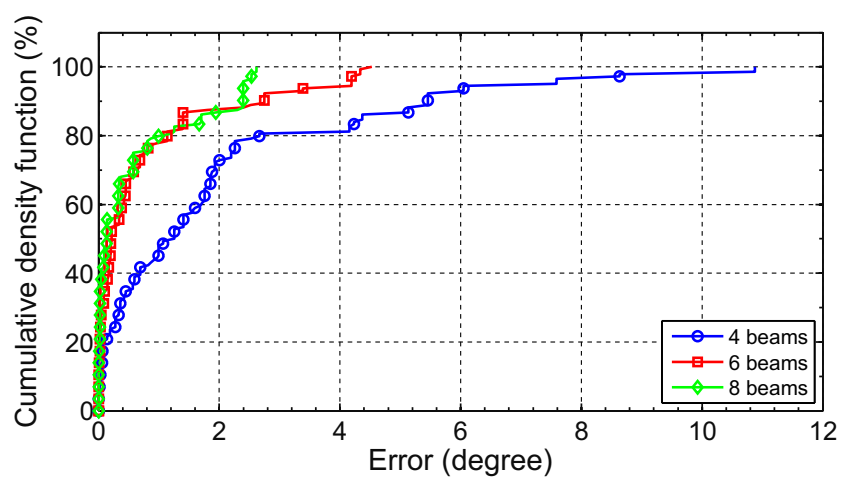

(b) Cumulative density function over $360^{\circ}$ angle

Fig. 4: Summary of the performance of the DoA algorithm applied to the data in Fig. 3.

Figure 4 shows the obtained results with the simulated data depicted in Fig. 3 after the calibration/equalization procedure. The estimation algorithm is applied to each test angle, and the resultant estimation error is depicted in Fig. 4a. In addition, Fig. 4b depicts the cumulative density error from the output data in Fig. 4a. Table I shows a comparative summary of the performance of the simulated cases. As expected, both the mean and the standard variation of the error decrease as the number of beams increases.

\section{EXPERIMENTAL VERIFICATION}

An experiment with the actual prototype depicted Fig. 1a was conducted. The prototype was already presented by the authors in [4], and it is based on the hardware described in section II. The 6 side antennas was used to fetch the RSSI 


\begin{tabular}{l|ccc}
\hline \hline case & mean error & max error & error std \\
\hline \hline 4 & 1.90 & 10.80 & 2.4 \\
\hline 6 & 0.75 & 4.50 & 1.20 \\
\hline 8 & 0.60 & 2.60 & 0.85 \\
\hline \hline $6(\exp )$ & 1.1 & 9.50 & 1.50 \\
\hline
\end{tabular}

TABLE I: A comparison of the results of the three simulated cases and the experimental case.

data reported in Fig. 5a. For each angle 10 RSSI sample was collected with the target antenna at $2 \mathrm{~m}$. As previously explained, the successful application of (11) is subjected to a preliminary calibration phase to adjust the theoretical quantity to the actual system characteristic, such as antenna gain imbalance, pointing angle and HPBW.

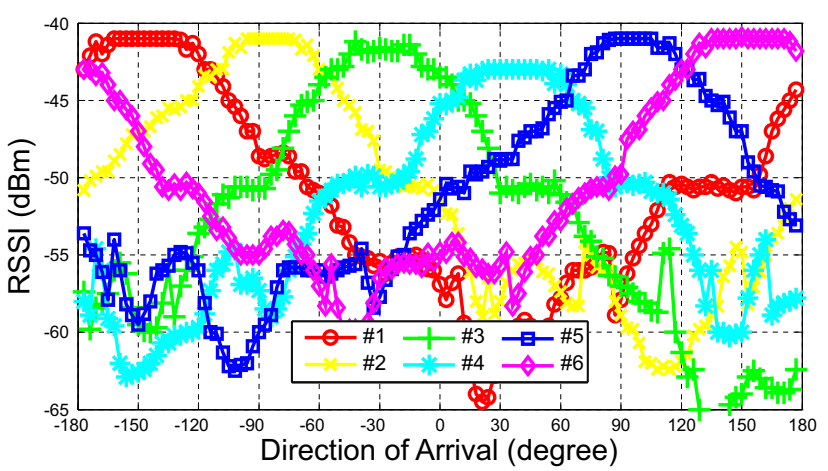

(a) Collected RSSI data.

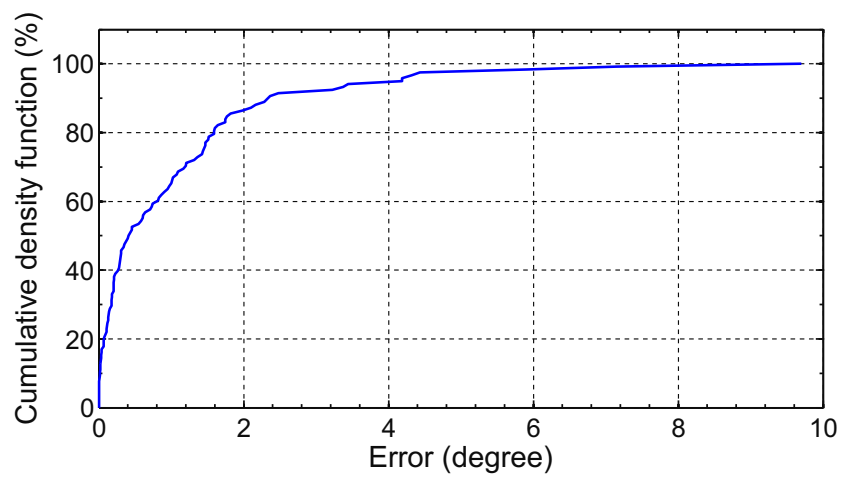

(b) Cumulative density function over $360^{\circ}$ angle.

Fig. 5: Experimental results operating with the 6SBA in Fig. 1a

Focusing on the data in Fig. 5a, the HPBW and pointing directions are quite matched with the simulated response for the case $\mathrm{N}=6$ : the principal source of error is the gain of the fourth beam. After a trivial calibration consisting in the adjustment of $G_{4}$, the DoA estimation results are depicted in Fig. $5 b$ in the form of a cumulative density function, which confirms the simulation results for the case $N=6$. In particular, a mean error of $1^{\circ}$ is obtained, with a STD of $1.6^{\circ}$, and a maximum error below $10^{\circ}$ (see Table I).

\section{CONCLUSIONS}

A novel algorithm for the estimation of Direction of Arrival with SBA was presented. The proposed algorithm relies only on a few fixed point closed-form calculations, therefore it is suitable for very low complexity hardware such as the industrial controller 8051. The proposed approach is based on the best analytic approximation of the pattern of a SBA and it is subsequent manipulation in the form of a quadratic equation. While simple, the proposed approach is robust enough to be used with real-life SBA node, operating with received signal strength indicator embedded within legacy IEEE 802.11 protocol. Tested with a set of computer generated data, the algorithm behaves as expected, with a max error of a few degrees for the case number of beam 4 , and decreasing for increasing beams. An experimental verification in a controlled anechoic setup confirms the validity of the proposed approach, demonstrating a good matching with the simulated data with a mean error of $1.1^{\circ}$ and a standard variation of $1.6^{\circ}$.

\section{REFERENCES}

[1] R. Zekavat and R. Buehrer, Handbook of Position Location: Theory, Practice and Advances. Wiley-IEEE Press, 2011, vol. 27.

[2] L. Bencini, S. Maddio, G. Collodi, D. Di Palma, G. Manes, and A. Manes, "Development of wireless sensor networks for agricultural monitoring," in Smart Sensing Technology for Agriculture and Environmental Monitoring. Springer, 2012, pp. 157-186.

[3] A. Cidronali, S. Maddio, G. Giorgetti, I. Magrini, S. Gupta, and G. Manes, "A $2.45 \mathrm{GHz}$ smart antenna for location-aware single-anchor indoor applications," in Microwave Symposium Digest, 2009. MTT'09. IEEE MTT-S International. IEEE, 2009, pp. 1553-1556.

[4] S. Maddio, M. Passafiume, A. Cidronali, and G. Manes, "A scalable distributed positioning system augmenting WiFi technology," in Indoor Positioning and Indoor Navigation (IPIN), 2013 International Conference on. IEEE, 2013, pp. 1-10.

[5] S. Maddio, A. Cidronali, and G. Manes, "Smart antennas for directionof-arrival indoor positioning applications," in Handbook of Position Location: Theory, Practice, and Advances. Wiley Online Library, 2011, pp. 319-355.

[6] M. Passafiume, S. Maddio, A. Cidronali, and G. Manes, "On the duality of Phase-based and Phase-less RSSI MUSIC algorithm for Direction of Arrival estimation," in 3rd International Conference on Circuits, Systems, Communications, Computers and Applications (CSCCA), Proceedings of. WSEAS, 2014, pp. 48-54.

[7] S. Maddio, M. Passafiume, A. Cidronali, and G. Manes, "Impact of the dihedral angle of switched beam antennas in indoor positioning based on rssi," in European Radar Conference (EuRAD), 2014 11th. IEEE, 2014, pp. 317-320.

[8] G. Giorgetti, S. Maddio, A. Cidronali, S. Gupta, and G. Manes, "Switched beam antenna design principles for angle of arrival estimation," in Wireless Technology Conference, 2009. EuWIT 2009. European. IEEE, 2009, pp. 5-8.

[9] R. Szumny, K. Kurek, and J. Modelski, "Attenuation of multipath components using directional antennas and circular polarization for indoor wireless positioning systems," in Radar Conference, 2007. EURAD 2007. European. IEEE, 2007, pp. 401-404.

[10] T. Rappaport and D. Hawbaker, "Wide-band microwave propagation parameters using circular and linear polarized antennas for indoor wireless channels," Communications, IEEE Transactions on, vol. 40, no. 2, pp. 240-245, 1992.

[11] R. Garg, Microstrip Antenna Design Handbook. Artech House, 2001.

[12] S. Maddio, "A Compact Wideband Circularly Polarized Antenna Array for C-band Applications," Antennas and Wireless Propagation Letters, IEEE, vol. 14, pp. 1081-1184, 2015.

[13] Y. Ozaki, J. Ozawa, E. Taillefer, J. Cheng, and Y. Watanabe, “A simple DOA estimator using adjacent pattern power ratio with switched beam antenna," in Wireless Communications and Signal Processing (WCSP), 2010 International Conference on. IEEE, 2010, pp. 1-6.

[14] S. Maddio, A. Cidronali, G. Giorgetti, and G. Manes, "Calibration of a $2.45 \mathrm{ghz}$ indoor direction of arrival system based on unknown antenna gain," in Radar Conference (EuRAD), 2010 European. IEEE, 2010, pp. $77-80$. 